\title{
Konzept zur Neuordnung der ärztlichen Bedarfsplanung
}

MARTIN ALBRECHT, HANS-DIETER NOLTING, ANKE SCHLIWEN

Dr. Martin Albrecht und Hans-Dieter Nolting sind Geschäftsführer des IGES Instituts in Berlin

Anke Schliwen ist wiss. Mitarbeiterin bei IGES

Die bisherige Bedarfsplanung hat dazu geführt, dass in ländlichen Regionen bei den meisten Arztgruppen eine viel geringere Arztdichte für angemessen erklärt wird als in den Ballungsgebieten und es auch innerhalb der Ballungsgebiete zu Ungleichverteilungen kommt. Mit dem im Jahr 2012 in Kraft getretenen Versorgungsstrukturgesetz wurde eine Reform der Bedarfsplanung angestoßen. Bis zum 1.1.2013 soll der Gemeinsame Bundesausschuss eine Neufassung der Bedarfsplanungsrichtlinie vorlegen. Das IGES Institut hat im Auftrag der Patientenvertretung im G-BA ein Konzept für die Neuordnung der ärztlichen Bedarfsplanung entwickelt. Es sieht vor, dass künftig Abweichungen der regionalen Arztdichte den Unterschieden des regionalen Versorgungsbedarfs entsprechen.

\section{Einleitung}

Die Bedarfsplanung legt fest, wie viele Einwohner ein niedergelassener Vertragsarzt normalerweise versorgen soll. Ein solches Normverhältnis wird gesondert für verschiedene ärztliche Fachgruppen bestimmt. Bei den NormVerhältniswerten handelt es sich um historische Einwohner-Arzt-Verhältnisse der 1990er Jahre für die betreffenden Arztgruppen in einem Regionstyp. Um festzustellen, ob ein Planungsbereich - typischerweise die kreisfreien Städte und Landkreise - als über-, angemessen oder unterversorgt einzustufen ist, werden die Norm-Verhältniswerte mit den tatsächlichen Einwohner-Arzt-Verhältnissen verglichen.

Anlass für eine Reform der Bedarfsplanung ist die zunehmend intensiv geführte gesundheitspolitische Diskussion über regionale Versorgungsunterschie- de. Im Mittelpunkt steht hierbei das teilweise ausgeprägte Gefälle des Zugangs zu niedergelassenen Ärzten: Demnach ist die Arztdichte in Ballungsgebieten bzw. Regionen mit vergleichsweise günstiger Sozialstruktur häufig deutlich höher als in ländlichen Regionen bzw. in Regionen mit vergleichsweise ungünstiger Sozialstruktur. Auch innerhalb von dicht versorgten Regionen kommt es zu Ungleichverteilungen, weil beispielsweise Praxen aus ärmeren Stadtteilen in die wohlhabenderen abwandern.

Im bestehenden Planungssystem werden Einwohnern in ländlichen Regionen verhältnismäßig deutlich weniger Ärzte zugestanden als Einwohnern in Städten und Ballungsgebieten. So soll ein Psychotherapeut in einer Kernstadt 2.577 Einwohner versorgen; in einem ländlichen Kreis hingegen mit 23.106 Einwohnern ungefähr die neunfache Anzahl.

Die bisherige Bedarfsplanung ver- 
hindert daher nicht, dass sich gerade dort lange Wartezeiten und Anfahrtswege ergeben können, wo der Versorgungsbedarf der gesetzlich Versicherten überdurchschnittlich hoch ist. Mit den existierenden Steuerungsmöglichkeiten zum Abbau von Über- und Unterversorgung kann solchen Problemen nur sehr begrenzt begegnet werden. Auch fehlt es der bisherigen Bedarfsplanung an $\mathrm{Zu}$ kunftsorientierung - sie berücksichtigt beispielsweise nicht die erwartete regionale Bevölkerungsentwicklung. Die gegenwärtigen Planungskriterien gelten als zu starr und undifferenziert - zu wenig orientiert am lokalen Bedarf und auch an den Versorgungskapazitäten angrenzender Sektoren.

Mit dem zum Jahresbeginn 2012 in Kraft getretenen GKV-Versorgungsstrukturgesetz (GKV-VStG) wurde die Weiterentwicklung der Bedarfsplanung angestoßen. Das Gesetz enthält vor allem allgemeine (Ziel-) Vorgaben und delegiert die erforderlichen Konkretisierungen in einer neuen BedarfsplanungsRichtlinie an den Gemeinsamen Bundesausschuss (G-BA). Die Neuregelungen des GKV-VStG bezüglich der Bedarfsplanung beziehen sich auf

- die Festlegung der Planungsbereiche (vgl. \ 101 Abs. 1 SGB V),

- die Bestimmung des Versorgungsbedarfs (vgl. \ 101 Abs. 2 SGB V) und

- die Feststellung des tatsächlichen Versorgungsangebots (vgl. \ 101 Abs. 1 Nr. 2a und Nr. 2b SGB V).

Zudem räumt das Gesetz der regionalen Ebene einen größeren Gestaltungsspielraum ein (vgl. $\int 99$ Abs. 1 SGB V).

Überwiegend bleiben die Neuregelungen der Bedarfsplanung im GKV-VStG abstrakt und bieten der Selbstverwaltung Spielraum bei der Ausgestaltung

\section{Ziel einer Reform sollte eine stärkere Orientierung am Versorgungsbedarf der regionalen Bevölkerung sein.}

\section{Bedarfsorientierung}

Eines der zentralen Ziele des IGESKonzepts für die Patientenvertretung im G-BA war die stärkere Orientierung der regionalen Planungswerte am Versorgungsbedarf der regionalen Bevölkerung. Zu diesem Zweck wurde ein Bedarfsindex entwickelt, der den Mehr- oder Minderbedarf für jede der kreisfreien Städte und jeden der Landkreise in Deutschland im Vergleich zum Bundesdurchschnitt beschreibt.

der künftigen Planung. Ob sich die Bedarfsplanung grundlegend verändert, dürfte auch davon abhängen, in welchem Verhältnis künftig zentrale Vorgaben des G-BA und die Entscheidungen
In den Bedarfsindex gehen nur Faktoren ein, für die ein Einfluss auf den Bedarf an ärztlichen Leistungen nachgewiesen werden kann und die weitgehend (statistisch) unabhängig vom bestehen- den ärztlichen Versorgungsangebot gemessen werden können. Dadurch wird das Risiko verringert, dass sich die Bedarfsplanung lediglich am dokumentierten Versorgungsbedarf orientiert und damit evtl. tatsächliche Versorgungsdefizite fortschreibt.

Für die Abbildung des Versorgungsbedarfs werden häufig drei Arten von Indikatoren verwendet:

- die demographische Struktur der Bevölkerung sowie die voraussichtliche demographische Entwicklung in einer Region ${ }^{1}$,

- Faktoren, die Rückschlüsse auf die Morbidität der regionalen Bevölkerung zulassen, sowie Morbiditätsprädiktoren ${ }^{2}$ und

- Faktoren, die sich aus der regionalen Sozialstruktur ergeben.

Auf Grundlage wissenschaftlicher Literatur und vorliegender empirischer Studien wurden die Indikatoren

- Alter und Geschlecht,

- Mortalität und Pflegebedürftigkeit und

- Arbeitslosigkeit und Einkommensarmut

\section{ausgewählt ${ }^{4}$.}

Der Bedarfsindex setzt sich zusammen aus den zwei Teilindices "Altersstruktur« und "Sozioökonomie / Morbidität«.

Für den Teilindex Altersstruktur wird der quantitative Zusammenhang zwischen der regionalen Altersstruktur und dem Versorgungsbedarf anhand

1 Vgl. u. a. Andersen \& Mühlbacher 2004.

2 Vgl. u. a. Birch et al. 1996, Newbold et al. 1998, Andersen \& Mühlbacher 2005.

3 Vgl. u. a. Mackenbach \& Maas 1989, Mielck 2000, Gibson et al. 2002, Helmert 2003, Lampert 2005, Maier et al. 2012, Czaja et al. 2012.

4 Als weitere bedarfsbestimmende Indikatoren wurden unter anderem auch die Anteile der Single-Haushalte, der Bevölkerung mit Migrationshintergrund, der Sozialhilfeempfänger und der Schulabgänger ohne und mit einfachem Bildungsabschluss geprüft. Aufgrund ihrer hohen Korrelationen mit den ausgewählten Indikatoren Arbeitslosigkeit, Einkommensarmut und Pflegebedarf wurden diese Indikatoren nicht in das Berechnungsmodell aufgenommen. Ihre zusätzliche Erklärungskraft ist so gering, dass kein signifikanter Informationsverlust dadurch entsteht, dass sie nicht unmittelbar bei der Bildung des Bedarfsindex berücksichtigt werden. 
des altersbezogenen durchschnittlichen Ausgabenprofils des Risikostrukturausgleichs (RSA) der GKV für ambulante Leistungen (in der bis 2008 geltenden Form des RSA) bestimmt. Die Abweichung des Versorgungsbedarfs einer Region aufgrund ihrer Altersstruktur wird ermittelt, indem ein altersstrukturgewichteter Durchschnitt der ambulanten GKV-Ausgaben für jede Region gebildet wird. Die prozentuale Abweichung dieses regionalen Wertes vom Bundesdurchschnitt entspricht dem Mehr- bzw. Minderbedarf an Ärzten einer Region allein aufgrund ihrer Altersstruktur. Dabei wird die relative Belastung durch die regionale Altersstruktur für Kinder und Erwachsene getrennt berechnet. Der Teilindex »Altersstruktur Kinder" bezieht sich auf die Altersgruppe 0-14 Jahre und wird für die Bedarfsplanung von Kinderärzten verwendet. Der Teilindex »Altersstruktur Erwachsene " bezieht sich auf die Bevölkerung im Alter ab 15 Jahren und findet bei allen anderen Fachgruppen Anwendung. ${ }^{5}$ Der Teilindex Altersstruktur entspricht somit streng genommen nicht unserem o. g. Kriterium der Unabhängigkeit der Bedarfsermittlung von den faktischen Versorgungsleistungen, da er sich vermittelt über das RSA-Profil letztlich auf abgerechnete vertragsärztliche Leistungen stützt. Im Hinblick auf die altersstrukturbedingte Variation des Bedarfs erscheint diese Endogenität jedoch vertretbar, da anzunehmen ist, dass das Altersprofil nur in geringem Maße von der regionalen Versorgungsangebotsdichte beeinflusst wird.

Im nächsten Schritt ist der quantitative Zusammenhang zwischen den Abweichungen der regionalen Ausprägung der ausgewählten sozioökonomischen und morbiditätsbezogenen Faktoren einerseits und dem regionalen Mehr- oder Minderbedarf an Ärzten andererseits zu bestimmen. Zunächst werden hierfür die drei Indikatoren Pflegebedarf, Arbeitslosigkeit und Einkommensarmut mithilfe einer Faktorenanalyse zu einem Wert verdichtet. Der Indikator Mortalität einer Region wird als Proxy-Variable für den Versorgungsbedarf verwendet und anhand des Kehrwerts der durchschnittlichen Lebenserwartung gebildet. Anhand einer Regressionsrechnung wird der Zusammenhang zwischen dem aus den Variablen Pflegebedarf, Arbeitslosigkeit und Einkommensarmut

Tabelle 1: Ergebnisse der Regressionsanalyse ( $n=412$ kreisfreie Städte und Landkreise)

\begin{tabular}{|l|c|c|c|c|c|}
\hline & \multicolumn{2}{|c|}{$\begin{array}{c}\text { Unstandardized } \\
\text { Coefficients }\end{array}$} & $\begin{array}{c}\text { Standar- } \\
\text { dized Coef- } \\
\text { ficients }\end{array}$ & t & \\
\cline { 2 - 5 } & Beta & $\begin{array}{c}\text { Std. } \\
\text { Error }\end{array}$ & Beta & & \\
\hline Konstante & 1,296 & 0,001 & & $1.433,519$ & 0,000 \\
\hline $\begin{array}{l}\text { Faktor } \\
\text { Morbidität / Sozio- } \\
\text { okonomie }\end{array}$ & 0,018 & 0,001 & 0,701 & 19,934 & 0,000 \\
\hline $\begin{array}{l}\text { Faktor } \\
\text { Alter und Geschlecht }\end{array}$ & 0,001 & 0,001 & 0,034 & 0,972 & 0,332 \\
\hline
\end{tabular}

a. Abhängige Variable: Kehrwert der Lebenserwartung 2008

Quelle: IGES

abgeleiteten Faktorscore (unabhängige Variable) und der Mortalität (abhängige Variable) ermittelt. Als Kontrollvariablen werden auch Alter und Geschlecht in die Regressionsgleichung aufgenommen.

Das Ergebnis der Regressionsanalyse zeigt einen signifikant positiven Zusammenhang zwischen dem Morbiditäts-/ Sozioökonomie-Faktor und der Mortalität (Tabelle 1). Mit dem Ergebnis kann für jede kreisfreie Stadt und jeden Landkreis die regionale Mortalität durch die regionalen Ausprägungen des Pflegebedarfs, der Arbeitslosigkeit und der Einkommensarmut geschätzt werden. Die prozentuale Abweichung dieser

\section{Der Mehr- oder Minderbedarf an Ärzten einer Region wird anhand eines Bedarfsindex bestimmt.}

regionalen geschätzten Mortalität vom Bundesdurchschnitt bildet den »Teilindex Morbidität / Sozioökonomie«.

Abschließend werden für jede kreisfreie Stadt und jeden Landkreis die Teilindices für Altersstruktur und Morbidität / Sozioökonomie gleichgewichtig $(0,5)$ addiert und somit zu einem Wert zusammengeführt. Durch die Gleichgewichtung können die durch die Teilindizes gemessenen Bedarfseinflüsse auch entgegengesetzt wirken. So kann ein Mehrbedarf einer überdurchschnittlich alten regionalen Bevölkerung durch einen Minderbedarf aufgrund ihrer günstigen Einkommenssituation und/ oder ihrer relativ geringen Morbidität ausgeglichen werden (und umgekehrt).
In der Zusammenschau aller 412 kreisfreien Städte und Landkreise ergibt sich eine Spreizung der Bedarfsabweichungen vom Bundesdurchschnitt

- für den Bedarfsindex »Kinder « zwischen $-2,1 \%$ bis $+4,6 \%$,

- für den Bedarfsindex "Erwachsene " zwischen $-4,2 \%$ bis $+4,7 \%$.

Dabei wurden die geringsten Werte für den Bedarfsindex »Kinder « für Landkreise in Nordrhein-Westfalen, Bayern und Baden-Württemberg ermittelt, die höchsten Werte für ostdeutsche Städte. Mit dem Bedarfsindex »Erwachsene « wurde ein stark unterdurchschnittlicher Bedarf in kreisfreien Städten und Landkreisen in Bayern, Baden-Württemberg und im westlichen Niedersachsen ermittelt; die höchsten Bedarfswerte wurden für kreisfreie Städte und Landkreise in östlichen Bundesländern und im östlichen Niedersachsen berechnet (Abbildung 1).

Für die Planung der spezialisierten fachärztlichen Versorgung sieht das IGES-Konzept eine Orientierung an größeren regionalen Einheiten vor, weil Versorgungskapazitäten stärker spezialisierter Fachärzte typischerweise aus Qualitäts- und Wirtschaftlichkeitsaspekten nicht flächendeckend wohnortnah vorgehalten werden können. Als

5 Sollte es nachweisbar abweichende Zusammenhänge zwischen Altersstruktur und Behandlungsaufwand für bestimmte Fachgruppen geben, kann die Ermittlung des Bedarfsindex entsprechend weitergehend differenziert werden. 
Planungsbereiche wurden die Raumordnungsregionen gewählt und analog zum beschriebenen Verfahren für jede Raumordnungsregion ein Bedarfsindex gebildet.

Der ermittelte Bedarfsindex wird im weiteren Bedarfsplanungsverfahren an zwei Stellen eingesetzt:

1. Die Regionen, für die ein mittlerer Versorgungsbedarf ermittelt wurde, bilden die Grundlage für die Ableitung der Norm-Verhältniszahlen.

2. Die prozentuale Abweichung vom Bundesdurchschnitt bildet den Mehroder Minderbedarf an ambulanter ärztlicher Leistung im Planungsbereich $\mathrm{ab}$.

Als Norm-Verhältniszahl für das ärztliche Angebot wird - differenziert nach Fachgruppe - die aktuelle Anzahl der Einwohner je Arzt herangezogen, die dem Durchschnitt der Regionen entspricht, für die ein mittlerer Versorgungsbedarf gemessen wurde. Die Norm leitet sich dabei aus dem Versorgungsgrad des »bedarfsmittleren Viertels « der Planungsbereiche ab. Dies gilt sowohl für die 412 kreisfreien Städte und Landkreise, als auch für die 96 Raumordnungsregionen. Die Ausrichtung am mittleren Viertel wurde als Kompromiss gewählt, um die Auswahl möglichst dicht am Bedarfsdurchschnitt zu halten und andererseits die Anfälligkeit der Auswahl gegenüber Regionen mit untypischer oder extremer Versorgungssituation möglichst gering zu halten. Der Normverhältniswert der Kinderärzte bezieht sich auf die Anzahl der Einwohner im Alter 0-14 Jahre, die Verhältniswerte der anderen Fachgruppen auf die Bevölkerung im Alter von 15 Jahren und älter.

Als Norm-Verhältniszahlen ergeben sich hieraus ein Sollwert von 1.368 Einwohnern im Alter von 15 Jahren und älter je Hausarzt und ein Sollwert von
2.055 Einwohnern im Alter von 0 bis 14 Jahren je Kinderarzt.

Die fachgruppenspezifischen NormVerhältniswerte gelten als einheitliche Planungsgrundlagen für alle kreisfreien Städte bzw. Landkreise bezüglich der wohnortnahen Versorgung und für alle Raumordnungsregionen bezüglich der spezialisierten fachärztlichen Versorgung. Eine Differenzierung nach Regionstyp wie im bestehenden System entfällt. Für jede Planungsregion wird die Soll-Ärztezahl anhand der regionalen Einwohnerzahl (Kinder oder Erwachsene) und der Norm-Verhältniszahl ermittelt. Der regionale Wert des Bedarfsindex bestimmt dann das Maß der Abweichung von der einheitlichen Planungsgrundlage (regionaler Mehroder Minderbedarf). Für Planungsbereiche mit einer überdurchschnittlichen Risikobelastung aufgrund der Alters-/ Geschlechtsstruktur, der Morbidität und der Sozialstruktur ergibt sich somit
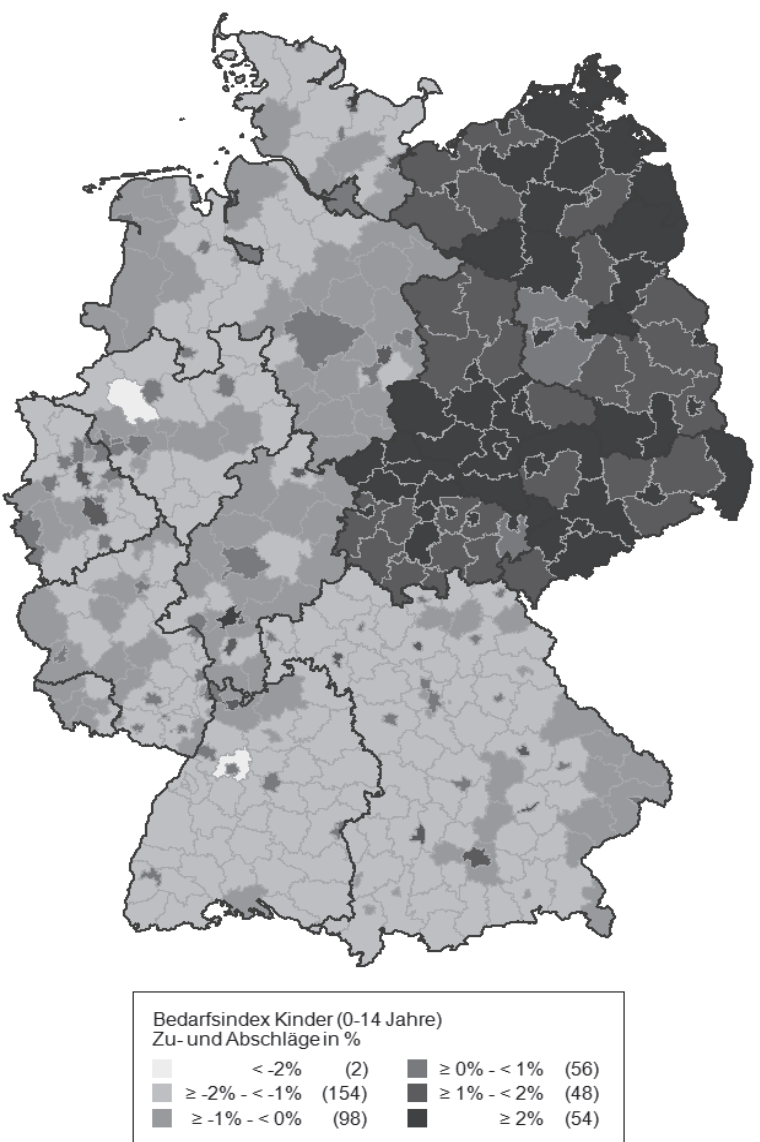

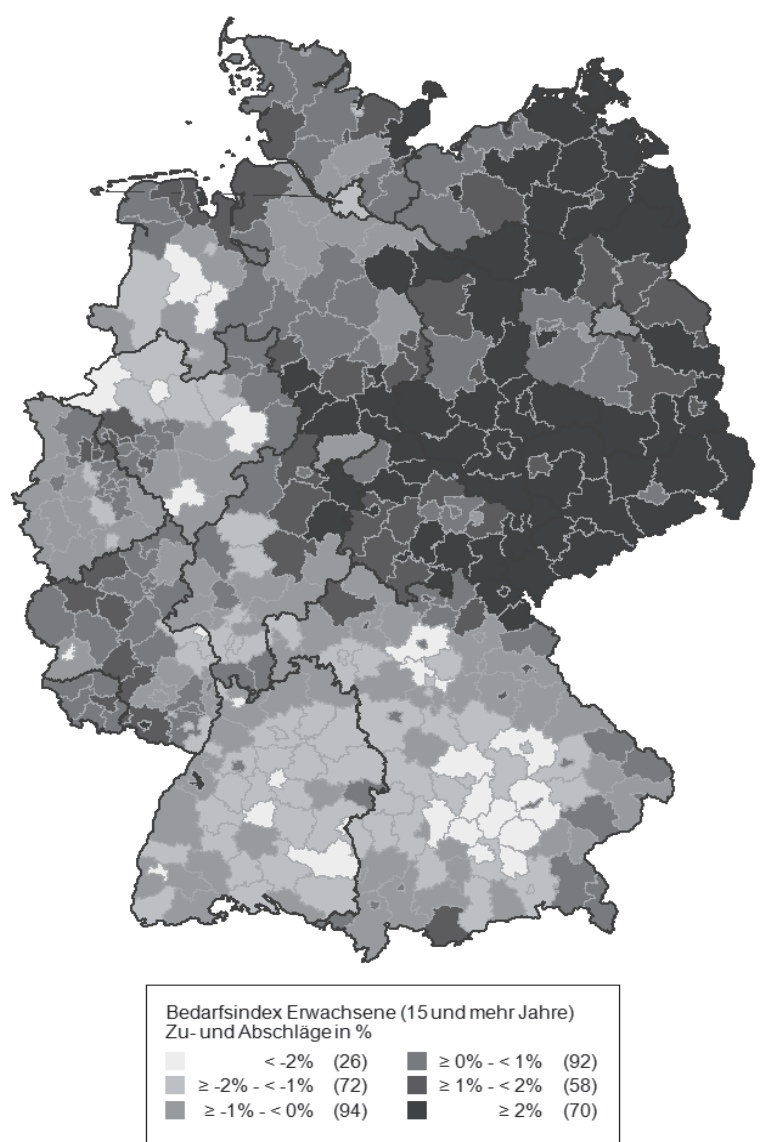


eine geringere Anzahl an Einwohnern, die von einem Arzt zu versorgen sind.

Eine so ermittelte neue Norm-Verhältniszahl auf Basis des »bedarfsmittleren Viertels « würde im Ergebnis ungefähr zu derselben Gesamtzahl an Hausärzten in der Bedarfsplanung führen. Allerdings ergäben sich teilweise sehr deutliche Verschiebungen zwischen den Regionen (Tabelle 2). So entstünden beispielsweise 161 zusätzliche Niederlassungsmöglichkeiten in Brandenburg, während der Hausärztebedarf in Berlin um 166 geringer veranschlagt würde.

Um zu prüfen, ob ein ermittelter Mehr- oder Minderbedarf an Ärzten auch langfristig Bestand hat, lässt sich auf Grundlage der Bevölkerungsvorausberechnungen der Statistischen Ämter der zukünftige Ärztebedarf für jede kreisfreie Stadt bzw. jeden Landkreis prognostizieren. Nach dem hier vorgeschlagenen Reformkonzept wird der prospektiv ermittelte Ärztebedarf fest in die Bedarfsplanung einbezogen. Dies betrifft insbesondere Situationen, in denen aktuell ein regionaler Mehrbedarf an Ärzten, längerfristig jedoch ein demographisch bedingter Rückgang des Ärztebedarfs ermittelt wird. Vorgeschlagen wird, einen auf Sicht von zehn Jahren nur als temporär festgestellten Mehrbedarf an Ärzten durch entsprechend befristete Zulassungen sowie ggf. andere Maßnahmen (z. B. mobile Arztpraxen, Patientenbeförderungsdienste) zu decken.

\section{Erreichbarkeit und weitere Faktoren als Bestandteil der Bedarfsplanung}

Ein weiterer Mangel der bisherigen Bedarfsplanung wird darin gesehen, dass die Erreichbarkeit von Arztpraxen kein explizites Planungskriterium ist. So ist es möglich, dass innerhalb eines Planungsbereichs die Arztsitze lokal sehr stark konzentriert sind und trotz einer insgesamt überdurchschnittlichen Arztdichte Teile der lokalen Bevölkerung nur begrenzten Zugang zur vertragsärztlichen Versorgung haben. Die gegenwärtige Rechtslage lässt eine weitergehende Arztsitzsteuerung nach dem Kriterium der Erreichbarkeit nicht zu. Eine Zulassungsmöglichkeit oder -sperre kann lediglich für einen Planungsbereich insgesamt ausgesprochen werden.

Das hier vorgelegte Konzept sieht

Tabelle 2: Abweichung der Anzahl Hausärzte (IST) von regionalen Planungswerten gemäß Reformkonzept (SOLL) nach Bundesländern

\begin{tabular}{|l|c|c|c|}
\hline & Anzahl IST & Anzahl SOLL & $\begin{array}{c}\text { Abweichung } \\
\text { SOLL ggü. IST }\end{array}$ \\
\hline Baden-Württemberg & 6.952 & 6.632 & $-4,6 \%$ \\
\hline Bayern & 8.845 & 7.773 & $-12,1 \%$ \\
\hline Berlin & 2.368 & 2.202 & $-7,0 \%$ \\
\hline Brandenburg & 1.494 & 1.655 & $10,8 \%$ \\
\hline Bremen & 448 & 425 & $-5,2 \%$ \\
\hline Hamburg & 1.240 & 1.110 & $-10,5 \%$ \\
\hline Hessen & 3.825 & 3.806 & $-0,5 \%$ \\
\hline Mecklenburg-Vorpommern & 1.118 & 1.092 & $-2,4 \%$ \\
\hline Niedersachsen & 4.853 & 4.980 & $2,6 \%$ \\
\hline Nordrhein-Westfalen & 10.795 & 11.203 & $3,8 \%$ \\
\hline Rheinland-Pfalz & 2.607 & 2.529 & $-3,0 \%$ \\
\hline Saarland & 647 & 662 & $2,3 \%$ \\
\hline Sachsen & 2.626 & 2.782 & $5,9 \%$ \\
\hline Sachsen-Anhalt & 1.437 & 1.584 & $10,2 \%$ \\
\hline Schleswig-Holstein & 1.889 & 1.787 & $-5,4 \%$ \\
\hline Thüringen & 1.431 & 1.495 & $4,5 \%$ \\
\hline insgesamt & $\mathbf{5 2 . 5 7 5}$ & $\mathbf{5 1 . 7 1 5}$ & $-\mathbf{1 , 6 \%}$ \\
\hline
\end{tabular}

Quelle: IGES; Ärztezahlen nach Kreisen (IST) gemäß Versorgungsatlas des ZI zum 31.12.2010

demgegenüber vor, die Kriterien Entfernung und Erreichbarkeit in die Bedarfsplanung aufzunehmen und bei der Verteilung der Praxisstandorte innerhalb eines Planungsbereiches zu berücksichtigen. Die absolute Sollzahl von Arztsitzen, die mithilfe der Normverhältniszahl und des Bedarfsindex für jeden Kreis festgelegt wurde, bleibt hiervon unberührt. Lediglich soll die Sollzahl von Arztsitzen in der Fläche so verteilt werden, dass Wohnortnähe und Erreichbarkeit für die regionale Gesamtbevölkerung optimiert werden können.

Eine solche Verteilungsplanung konzentriert sich vor allem auf diejenigen Arztgruppen, deren Leistungsangebot wohnortnah vorgehalten werden soll, wobei sich das anzuwendende Entfernungskriterium je nach Arztgruppe unterscheiden kann. Im IGES-Konzept wurden $5 \mathrm{~km}$ für die Entfernung zur nächsten Hausarztpraxis und $15 \mathrm{~km}$ für die wohnortnahe fachärztliche Versorgung (z. B. Augenärzte, Gynäkologen, Orthopäden) verwendet.

Die Verteilungsplanung erfolgt auf Ebene der Gemeinden in Landkreisen und auf Ebene der Stadt-/Ortsteile in kreisfreien Städten. In einem ersten Schritt ermittelt die zuständige KV die bestehenden Praxisstandorte und berechnet die faktischen EinwohnerArzt-Verhältnisse auf Gemeindeebene. Die Gemeinden werden dann einer von vier Kategorien zugeordnet:

A. Gemeinden, in denen die faktische Einwohner-Arzt-Relation der Zielrelation des Kreises entspricht $(75 \% \leq$ Zielrelation $\leq 125 \%$ )

B. Gemeinden mit einer höheren Versorgungsdichte als Kategorie A.

C. Gemeinden mit einer geringeren Versorgungsdichte als Kategorie A.

D. Gemeinden ohne Praxisstandort

Für Gemeinden der Kategorie D (ohne Praxisstandort) wird anschließend geprüft, ob ihre Bevölkerung durch Arztstandorte in benachbarten Gemeinden mitversorgt werden kann, indem die jeweiligen Gemeinden fiktiv vereinigt werden. Dabei muss gewährleistet sein, dass die Einwohner-Arzt-Relation nach der Zusammenfassung noch innerhalb 


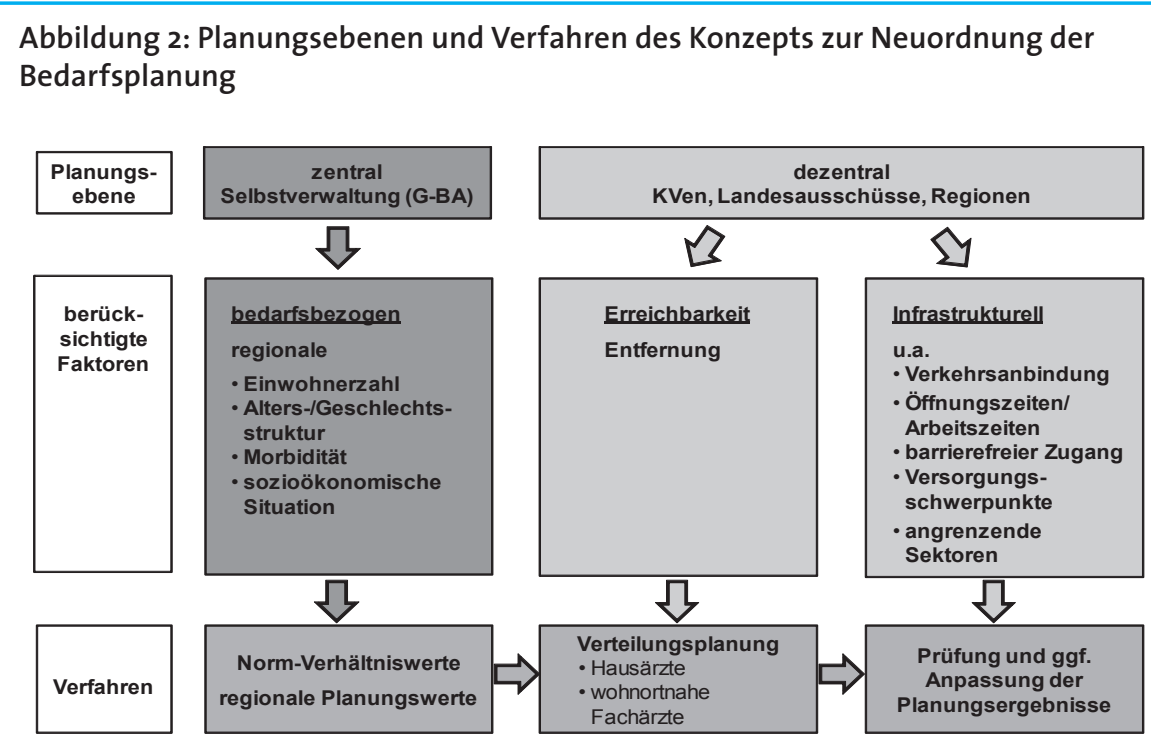

Quelle: IGES

einbart werden, dass frei werdende Arztsitze in Kategorie-B-Gemeinden nur mit der Auflage vergeben werden, den Standort in eine Kategorie-D- bzw. C-Gemeinde zu verlegen.

Die Landesausschüsse sollen bei der Verteilungsplanung neben den lebensweltlichen und verkehrstechnischen Verflechtungen auch weitere Faktoren einbeziehen, die den lokalen Zugang zur ärztlichen Versorgung beeinflussen können. So können einzelne Arztpraxen aufgrund kurzer Praxisöffnungszeiten, Spezialisierung ihres Versorgungsangebots (bspw. Augenärzte, die sich auf Katarakt-Operationen spezialisiert haben) oder fehlender Barrierefreiheit nur mit Einschränkungen für die Versorgung der Versicherten zur Verfügung stehen. Andererseits können Versorgungsangebote angrenzender Sektoren (z. B. Krankenhausambulanzen) mögliche

der Intervallgrenzen $75 \%-125 \%$ Versorgungsgrad liegt und dass die Einwohner der D-Gemeinden die Ärzte in der benachbarten Gemeinde in zumutbarer Entfernung erreichen können $(\leq 5 \mathrm{~km}$ für Hausärzte, $\leq 15 \mathrm{~km}$ für wohnortnahe Fachärzte). Falls eine Zusammenfassung nach diesen Bedingungen nicht in Betracht kommt, ist ein zusätzlicher Arztsitz in der D-Gemeinde zu planen. Ebenso wird mit Gemeinden der C-Kategorie verfahren. Das Ergebnis dieser Einzelprüfungen der Gemeinden in einem Planungsbereich ist eine Liste von Gemeinden, die für einen neuen Praxisstandort in Betracht kommen. Würde durch die Anzahl zusätzlicher Arztsitze die Soll-Zahl für den Planungsbereich insgesamt überschritten, ist zu entscheiden, für welche Gemeinden prioritär ein zusätzlicher Praxisstandort geplant werden soll. Das Konzept sieht diese Priorisierung als Aufgabe der KV vor, die hierbei das lokale Wissen über die lebensweltlichen und verkehrstechnischen Verflechtungen und Gegebenheiten in der Region nutzen soll. Dabei wird bspw. geprüft, ob eine Überschreitung der Maximalentfernung zur nächsten Praxis durch eine vergleichsweise günstige Verkehrsanbindung ausgeglichen werden kann. Ziel der Priorisierung ist es, eine möglichst geringe Abweichung von der Zielrelation in möglichst vielen Gemeinden zu erreichen.

Für Gemeinden der Kategorie B ist die umgekehrte Vorgehensweise bei der Standortplanung anzuwenden. Hierzu Versorgungsmängel im vertragsärztlichen Sektor ausgleichen.

ist das Ausmaß der Mitversorgerfunktion der Standorte in Kategorie-B-Gemeinden zu ermitteln und festzustellen, ob nach Wegfall eines Standortes innerhalb der Gemeinde sowie bei Zusammenfassung mit ihren Nachbarn die Zielrelation des Kreises erhalten bleibt. Nach dieser Prüfung ergibt sich eine zweite Liste mit Standorten, die potentiell wegfallen können.

Auch die Gemeinden in denen die faktische Einwohner-Arzt-Relation der Zielrelation des Kreises entspricht, sollen im Hinblick auf die Wohnortnähe der Standorte geprüft werden. Eine perspektivische Umsiedlung von Standorten kann auch in Gemeinden notwendig sein, in denen die Zielrelation erreicht ist.

Die perspektivische Verlegung von Praxisstandorten zur Herstellung einer größeren Wohnortnähe ist eine lang-

\section{Die Verteilung der Praxis- standorte berücksichtigt die Entfernung und weitere Faktoren des lokalen Zugangs zum Arzt.}

\section{Umsetzung und Gestaltung des Planungsprozesses}

Die Umsetzung der hier skizzierten Bedarfsplanung mit den drei Verfahrensstufen

1. Bestimmung der Norm-Verhältniszahlen und der regionalen Planungswerte,

2. Kapazitätsverteilung in der Fläche (Verteilungsplanung),

3. Prüfung und ggf. Anpassung der Planungsergebnisse unter Berücksichtigung weiterer Faktoren des Zugangs zur ärztlichen Versorgung

soll verschiedenen Planungsebenen zugeordnet werden. Die Norm-Verhältniszahlen und die regionalen Planungswerte sollen nach bundeseinheitlichen Verfahren auf zentraler Ebene der Selbstverwaltung durch den G-BA bestimmt werden. Die Aufstellung der Bedarfspläne und die Verteilungsplanung von Arztstandorten innerhalb der Planungsbereiche würden

fristige Rahmenplanung, die nicht auf der Umsiedlung von Vertragsärzten mit ihrer Praxis beruht, sondern auf der Verlegung von Praxisstandorten ausschließlich im Falle einer Nachbesetzung des Arztsitzes. So kann beispielsweise ver- hingegen auf dezentraler bzw. regionaler Ebene durchgeführt (Abbildung 2).

Das vorgeschlagene Verfahren basiert auf dem Grundsatz, lokales Wissen auch auf lokaler Ebene im Planungsprozess einfließen zu lassen. Damit soll eine 
hohe Effizienz der Informationsbeschaffung und -verwertung erreicht werden. Gleichzeitig wird damit dem Ziel entsprochen, die Bedarfsplanung stärker regional $\mathrm{zu}$ verankern und regionale Besonderheiten zu berücksichtigen.

Für die Legitimität des Verfahrens erscheint es notwendig, für alle Versicherten der GKV die gleichen Grundsätze der Messung und Berechnung des regionalen Bedarfs zugrunde zu legen. Daher ist die Selbstverwaltung auf Bundesebene der geeignete Ort für die Festlegung der Norm-Verhältniswerte sowie der regionalen Planungswerte.

Die weiteren Faktoren der Bedarfsplanung beziehen sich auf den lokalen Zugang zu Versorgungsleistungen. Informationen und Kenntnisse über verkehrstechnische Gegebenheiten (z. B. ÖPNV) und die Charakteristika des lokalen Versorgungsangebots (Öffnungszeiten, barrierefreier Zugang, Versorgungsschwerpunkte, Zugang zu Versorgungsangeboten angrenzender Sektoren etc.) sind hingegen auf regionaler bzw. lokaler Ebene leichter verfügbar. Eine zentrale Beschaffung und Administration dieser Informationen wäre zwar prinzipiell möglich, ist aber mit großem Aufwand verbunden. $\mathrm{Zu}$ -

\section{Für alle GKV-Versicherten müssen die gleichen Grundsätze der Messung und Berechnung des regionalen Bedarfs zugrunde gelegt werden.}

dem soll die Auswahl der konkret zu betrachteten infrastrukturellen Faktoren regional bzw. fachbezogen auf bestimmte Planungsbereiche konzentriert werden anstatt sämtliche Informationen für alle Planungsbereiche zu verarbeiten, wie es in einem zentralisierten Verfahren der Fall wäre.

\section{Fazit}

Ziel der Bedarfsplanung ist es, die Kapazitäten und Zugangsmöglichkeiten einer gegebenen Gesamtversorgung regional zu verteilen. Vor dem Hintergrund der zunehmenden Kritik an den Mängeln der bisherigen Bedarfsplanung sowie der durch das GKV-Versorgungsstrukturgesetz angestoßenen Reform der Bedarfsplanung hat das IGES Institut im Auftrag der Patientenvertretung im G-BA ein Reformkonzept entwickelt. Kernelemente dieses Konzepts sind

v eine stärkere Orientierung der Planung von Arztsitzen am regionalen Versorgungsbedarf,

- die Berücksichtigung der Erreichbarkeit von Ärzten und

- eine Verbesserung der regionalen Gestaltungsmöglichkeiten zur Deckung des Versorgungsbedarfs.

Zur Bestimmung des regionalen Versorgungsbedarfs wurde auf der Basis demographischer, morbiditätsbezogener und sozioökonomischer Faktoren ein Bedarfsindex entwickelt. Regionen mit einem mittleren Bedarf bestimmen das Norm-Verhältnis von Einwohnern je Arzt. Die regionalen Planungswerte variieren ausschließlich nach den ermittelten Bedarfsunterschieden - und nicht mehr wie bisher nach Regionstyp. Die zukünftige Bevölkerungsentwicklung ist in dem Konzept fester Bestandteil der Planung. Darüber hinaus ist eine regionale Verteilungsplanung von Arztstandorten vorgesehen, um eine optimale Wohnortnähe und Erreichbarkeit der ärztlichen Versorgung zu ermöglichen. In diesem Zusammenhang wird eine stärkere Einbeziehung der regionalen Ebene in die Bedarfsplanung vorgeschlagen.

\section{Literatur}

Andersen HH, Mühlbacher A (2004): Bedarfsplanung und demographische Entwicklung. Zum Einfluß der Bevölkerungsstruktur auf die hausärztliche Versorgung. Berliner Zentrum Public Health, Blaue Reihe.

Andersen HH, Mühlbacher A (2005): Morbidität, Bedarf und Vergütung. Methodische Grundlagen für Analysen über Zusammenhänge in der ambulanten Versorgung. Das Beispiel morbiditätsorientierter, regionalisierter Prognosen des Ärztebedarfs. Gesundheit und Sozialpolitik, 5/6: 20-30.

Birch S, Eyles J, Newbold KB (1996): Proxies for health care need among populations: a validation of alternatives - a study in Quebec. Journal of Epidemiology and Community Health 50(5): 564-569.

Czaja M, Meinlschmidt G, Bettge S (2012): Sozialindikative Planung der regionalärztlichen Versorgung - Ein Diskussionsbeitrag für Berlin am Beispiel der Psychotherapeuten und Hausärzte. Gesundheit- und Sozialpolitik 3: 34-43.

Gibson A, Asthana S, Brigham P, Moon G, Dicker J (2002): Geographies of need and the new NHS: methodological issues in the definition and measurement of the health needs of local populations. Health \& Place 8: 47-6o.

Helmert U (2003): Soziale Ungleichheit und Krankheitsrisiken. Beiträge zur Sozialpolitikforschung. Augsburg: Marco Verlag.

Lampert, T., Saß, A-C, Häfelinger, M. und Ziese, T. (2005): Armut, soziale Ungleichheit und Gesundheit. Expertise des Robert KochInstituts zum 2. Armuts- und Reichtumsbericht der Bundesregierung (Beiträge zur Gesundheitsberichtserstattung des Bundes).

Maier, W., Fairburn, J. und Mielck, A. (2012): Regionale Deprivation und Mortalität in Bayern. Entwicklung eines IIndex Multipler Deprivation، auf Gemeindeebene. Das Gesundheitswesen 74(07): 416-425.

Mackenbach JP, Maas PJ (1989): Social inequality and differences in health: A survey of the principal research findings. In: Gunning-Schepers LJ, Spruit IP, Krijnen JH (Ed.) Socio economic inequalities in health questions on trends and explanations. Den Haag: $25-80$

Mielck A (2000): Soziale Ungleichheit und Gesundheit: Empirische Ergebnisse, Erklärungsansätze, Interventionsmöglichkeiten. Bern: H. Huber.

Newbold KB, Eyles J, Birch S, Spencer A (1998): Allocating resources in health care: alternative approaches to measuring needs in resource allocation formula in Ontario. Health \& Place 4(1): 79-89. 